University of Nebraska - Lincoln

DigitalCommons@University of Nebraska - Lincoln

9-8-1998

\title{
Validity of Tolman's equation: How large should a droplet be?
}

\author{
Kenichiro Koga \\ University of Nebraska-Lincoln, koga@cc.okayama-u.ac.jp \\ Xiao Cheng Zeng \\ University of Nebraska-Lincoln, xzeng1@unl.edu
}

A.K. Shchekin

St. Petersburg State University

Follow this and additional works at: https://digitalcommons.unl.edu/chemzeng

Part of the Chemistry Commons

Koga, Kenichiro; Zeng, Xiao Cheng; and Shchekin, A.K., "Validity of Tolman's equation: How large should a droplet be?" (1998). Xiao Cheng Zeng Publications. 65.

https://digitalcommons.unl.edu/chemzeng/65

This Article is brought to you for free and open access by the Published Research - Department of Chemistry at DigitalCommons@University of Nebraska - Lincoln. It has been accepted for inclusion in Xiao Cheng Zeng Publications by an authorized administrator of DigitalCommons@University of Nebraska - Lincoln. 


\title{
Validity of Tolman's equation: How large should a droplet be?
}

\author{
Kenichiro Koga ${ }^{\text {a) }}$ and X. C. Zeng \\ Department of Chemistry and Center for Materials Research and Analysis, University of Nebraska, Lincoln, \\ Nebraska 68588 \\ A. K. Shchekin \\ Department of Statistical Physics, St. Petersburg State University, 198904, St. Petersburg, Russia
}

(Received 23 February 1998; accepted 2 June 1998)

\begin{abstract}
Surface tension and the length $\delta$ (distance between the Gibbs surface of tension $R_{s}$ and the equimolar surface $R_{e}$ ) of simple liquid droplet (Lennard-Jones and Yukawa) are computed over a wide range of droplet sizes up to about $4 \times 10^{6}$ molecules. The study is based on the Gibbs theory of capillarity combined with the density-functional approach to gas-liquid nucleation. Since this method provides behavior of the surface tension fully consistent with the tension of the planner surface, the constant in Tolman's equation $\delta_{\infty}$ can be determined unequivocally from the asymptotic behavior of $\sigma_{s}$. Comparison of the tension given by Tolman's equation against the result of exact thermodynamic relations reveals that Tolman's equation is valid only when the droplet holds more than $10^{6}$ molecules for the simple fluid systems near their triple points, in contrast to the conventional wisdom that Tolman's equation may be applicable down to droplets holding a few hundreds of molecules. (C) 1998 American Institute of Physics. [S0021-9606(98)50334-0]
\end{abstract}

\section{INTRODUCTION}

One fundamental question on properties of droplets of liquid is the dependence of surface tension on droplet radius. Numerous theoretical investigations have been devoted to this question, including the original thermodynamic consideration by Gibbs, ${ }^{1}$ later development by Tolman, ${ }^{2}$ and recent studies based on statistical mechanical theories ${ }^{3-8}$ and computer simulations. ${ }^{9-12}$

When a droplet is sufficiently large, size dependence of the surface tension can be expressed by Tolman's equation of the form ${ }^{2}$

$$
\frac{\sigma_{s}}{\sigma_{\infty}}=\frac{R_{s}}{R_{s}+2 \delta_{\infty}} .
$$

Here, $\sigma_{s}$ is the surface tension with respect to the Gibbs surface of tension and $R_{s}$ denotes the radius of this dividing surface; $\sigma_{\infty}$ is the planner surface tension, and $\delta_{\infty}$ is defined as $\lim _{R_{s} \rightarrow \infty} \delta$, where $\delta$ is the distance between the equimolar surface with radius $R_{e}$ and the surface of tension. Tolman derived Eq. (1) based on the Gibbs theory of capillarity ${ }^{1}$ plus two additional assumptions: $\delta \ll R_{s}$ (assumption $\mathrm{A}$ ) and $\delta$ $=\delta_{\infty}$ (assumption B).

On the other hand, in the case of small droplets, no analytical relation has been found so far to express $\sigma_{s}$ in terms of $R_{s}$. There have been several numerical calculations of $\sigma_{s}$ for various sizes of droplets via the gradient theories, ${ }^{3,4,6}$ the density-functional theory, ${ }^{7}$ and computer simulations. ${ }^{9-12}$ Most studies reached a common conclusion that very rapid decrease in surface tension takes place with decrease in droplet size when $R_{S}$ is sufficiently small (e.g., smaller than about ten molecular radii ${ }^{3}$ ). Some studies ${ }^{3,7}$ also showed that $\delta$ is a

${ }^{\text {a)} E l e c t r o n i c ~ m a i l: ~ k o g a @ ~ p h a s e 2 a . u n l . e d u ~}$ strong function of $R_{s}$, i.e., assumption B is invalid for very small droplets. ${ }^{13}$ Size dependence of surface tension and length $\delta$ becomes more and more controversial as one considers larger and larger droplets. This is mainly due to the fact that numerical errors in the calculations overwhelm the precision one needs for large droplets for which dependence of $\sigma_{s}$ and $\delta$ on $R_{s}$ is very weak. To our knowledge, no quantitative information has been reported as to a range of droplet sizes where we can apply Tolman's equation for calculating surface tension.

The purpose of this article is to shed some light on to what extent Tolman's equation is valid. To this end, we compute the surface tension and the length $\delta$ for a wide range of droplet sizes on the basis of the Gibbs thermodynamic theory of capillarity combined with the density-functional theory of gas-liquid nucleation. ${ }^{14,15}$

We first study behavior of $\delta$ for sizes of droplet ranging from 40 to about $4.0 \times 10^{6}$ particles. Two different routes to $\delta$, both of which are exact, are taken: a direct route which calculates $R_{e}$ and $R_{s}$ separately, and an adsorption route we propose in Sec. IV. We will show that the direct route gives rise to unphysical behavior of $\delta$ when we approach the planner surface limit whereas the adsorption route can lead to self-consistent and precise results of asymptotic behavior of the length $\delta$. From the asymptotic behavior, the value of Tolman's length $\delta_{\infty}$ can be determined accurately. It is then possible to examine the range of droplet sizes where Tolman's equation is valid by comparing results of Tolman's equation (using $\delta_{\infty}$ ) with those from rigorous calculations based on the thermodynamic definition of surface tension.

We also discuss the validity of other thermodynamic routes (mostly used in computer simulations) to surface tension, length $\delta$, and Tolman's length $\delta_{\infty}$. One such route is the thermodynamic route proposed by Thompson et al. ${ }^{10}$ in the molecular dynamics simulation study of Lennard-Jones 
(LJ) liquid drops. Recently, Nijmeijer et al. ${ }^{11}$ adopted another thermodynamic route to $\delta$ in large scale computer simulations. Both of the routes seem particularly convenient for the computer simulation because they require neither the equation of state of the bulk fluid nor the grand potential of the droplet-vapor system. We note, however, that these routes are based on several approximations to the rigorous thermodynamic theory and become exact only in the large droplet limit. We therefore also examined the extent to which these routes are applicable to the determination of $\sigma_{s}$ and $\delta$.

This paper is organized as follows: Section II gives Gibbs thermodynamic description of the droplet-vapor system. Section III summarizes the density-functional theory of gas-liquid nucleation. Results and discussion are given in Sec. IV and concluding remarks are given in Sec. V.

\section{THERMODYNAMIC DESCRIPTION OF THE DROPLET-VAPOR SYSTEM}

We consider a critical droplet of a single-component liquid in (unstable) equilibrium with a supersaturated vapor at temperature $T$. The system is taken to be a conical section (with solid angle $\omega$ ) of a sphere (with radius $\mathscr{R}$ ) which includes the droplet at the center with surrounding vapor. The radius $\mathscr{B}$ is chosen to be sufficiently large such that the vapor at the boundary acquires the bulk properties. The chemical potential $\mu$ of the system is higher than the chemical potential $\mu_{\text {eq }}$ at vapor-liquid (stable) equilibrium at $T$. The reversible transformations of the system are described by the Gibbs fundamental relation

$$
d U=T d S-d W+\mu d N,
$$

where the symbols $U, S$ and $N$ denote, respectively, the energy, entropy and number of molecules of the system. The work performed by the system is represented by $d W$. To define the surface or excess variables including surface tension, one needs to introduce a mathematical dividing surface with an arbitrary radius $R$, which partitions the total volume $(V)$ into two parts: one inside the dividing surface $V^{\alpha}$ and the other part $V^{\beta}$. Then a hypothetical reference system is introduced, which possesses the same $\mu, T$, and $V$ but is composed of the uniform liquid and vapor phases (let us call them $\alpha$ and $\beta$ phases, respectively) right up to the dividing surface; namely, the reference system consists of two parts: a portion $\left(V^{\alpha}\right)$ of a bulk liquid at $\mu$ and $T$ and a portion $\left(V^{\beta}\right)$ of a bulk gas at $\mu$ and $T$. Since $\mu>\mu_{\text {eq }}$, phase $\alpha$ is thermodynamically stable, whereas phase $\beta$ is metastable. Now the surface variables can be defined as

$$
\begin{aligned}
& N^{s}=N-N^{\alpha}-N^{\beta}, \\
& U^{s}=U-U^{\alpha}-U^{\beta}, \\
& S^{s}=S-S^{\alpha}-S^{\beta},
\end{aligned}
$$

where the superscripts $\alpha$ and $\beta$ indicate the quantities of each part of the reference system. The infinitesimal change in the internal energy of each part of the reference system is described by the fundamental relation

$$
d U^{\alpha}=T d S^{\alpha}-p^{\alpha} d V^{\alpha}+\mu d N^{\alpha},
$$

$$
d U^{\beta}=T d S^{\beta}-p^{\beta} d V^{\beta}+\mu d N^{\beta} .
$$

It is important to emphasize that $p^{\alpha}$ is not the pressure $p(0)$ at the center of the droplet but is the pressure of homogeneous phase $\alpha$ in the reference system; in general, these two values are different. The change in the surface energy is assumed to be given by

$$
d U^{s}=T d S^{s}+\sigma d A+C d R+\mu d N^{s},
$$

where $A$ is the surface area of the dividing surface and $\sigma$ is the surface tension defined as a conjugate variable of $A ; C$ is the conjugate variable of $R$. Combining Eqs. (6)-(8) results in a relation

$$
d U=T d S-p^{\alpha} d V^{\alpha}-p^{\beta} d V^{\beta}+\sigma d A+C d R+\mu d N .
$$

Comparing Eqs. (9) with (2), one can find that $\sigma d A$ $+C d R$ is the difference between the work done on the real system $(-d W)$ and the work done on the hypothetical reference system $\left(-p^{\alpha} d V^{\alpha}-p^{\beta} d V^{\beta}\right)$. Integrating Eq. (9) with respect to the solid angle $\omega$ at fixed $T, \mu$ and $R$ yield

$$
\sigma=\frac{\Omega+p^{\alpha} V^{\alpha}+p^{\beta} V^{\beta}}{A}=\frac{\Delta \Omega+\Delta p V^{\alpha}}{A},
$$

where $\Omega \equiv U-T S-\mu N$ is the grand potential, $\Delta \Omega \equiv \Omega$ $+p^{\beta} V$ is the work of formation of the critical droplet, and $\Delta p$ stands for $p^{\alpha}-p^{\beta}$. Equation (10) holds for surface tension with respect to any dividing surface. Note that $\sigma$ depends not only on the thermodynamic state of the system but also on the choice of the parameter $R$. However, if a particular dividing surface such as the surface of tension or the equimolar surface is chosen, the corresponding surface tension turns out to be a state function.

The surface of tension with radius $R_{s}$ is a special dividing surface for which the coefficient $C$ in Eq. (9) vanishes and the Laplace equation holds: ${ }^{16}$

$$
\Delta p=2 \sigma_{s} / R_{s} .
$$

Recalling that $V^{\alpha}=4 \pi R_{s}^{3} / 3$ and $A=4 \pi R_{s}^{2}$, one can solve the coupled equations (10) and (11) to obtain

$$
\sigma_{s}=\left(\frac{3 \Delta \Omega(\Delta p)^{2}}{16 \pi}\right)^{1 / 3}
$$

and

$$
R_{s}=\left(\frac{3 \Delta \Omega}{2 \pi \Delta p}\right)^{1 / 3} .
$$

These are rigorous thermodynamic expressions for $\sigma_{s}$ and $R_{s}$. One may question the validity of thermodynamic arguments when a droplet is too small to possess bulk liquid properties inside its surface. However, as noted by Gibbs, ${ }^{1}$ his original thermodynamic arguments (presented above) do not assume any thermodynamic property inside the droplet; instead they rely upon the properties of the homogeneous liquid phase having the same chemical potential as the droplet-vapor system has. Whether or not there is a homogeneous liquid phase inside the droplet, the thermodynamic quantities of the reference system are well defined. Therefore, the surface tension and other surface quantities are well 
defined no matter how small the droplet is. In this sense, the Gibbs thermodynamic arguments hold for any size of a droplet. On the other hand, any thermodynamic arguments relying upon thermodynamic quantities in the actual droplet (e.g., pressure at the center of the droplet) become less and less reliable when we go to smaller and smaller droplets.

Tolman's equation is derived as follows. From the Gibbs-Duhem equation for the surface variables ( $A d \sigma_{s}$ $\left.+N^{s} d \mu+S^{s} d T=0\right)$ together with the definition of the surface adsorption $\left(\Gamma_{s} \equiv N^{s} / A\right)$, we have

$$
\Gamma_{s}=-\left(\partial \sigma_{s} / \partial \mu\right)_{T}
$$

Combining a similar relation for the reference system, $\Delta \rho$ $\equiv \rho^{\alpha}-\rho^{\beta}=(\partial \Delta p / \partial \mu)_{T}$, with Eq. (14) yields a differential equation:

$$
\left(\frac{\partial \sigma_{s}}{\partial \Delta p}\right)_{T}=-\frac{\Gamma_{s}}{\Delta \rho} .
$$

In the planner surface limit, the quantity $\Gamma_{s} / \Delta \rho$ on the right hand side of Eq. (15) is identical to Tolman's length $\delta_{\infty}$. This is seen from an exact relation (originally derived by Tolman ${ }^{2}$ )

$$
\frac{\Gamma_{s}}{\Delta \rho}=\delta\left(1+\frac{\delta}{R_{s}}+\frac{1}{3} \frac{\delta^{2}}{R_{s}^{2}}\right) .
$$

If we integrate Eq. (15) with respect to $\Delta p$ from the planar surface limit $(\Delta p=0)$, assuming that $\Gamma_{s} / \Delta \rho$ is constant over the range, we find

$$
\sigma_{s}-\sigma_{\infty}=-\delta_{\infty} \Delta p .
$$

Substitution of Laplace equation (11) into Eq. (17) results in Tolman's equation (1).

\section{APPLICATION OF THE DENSITY-FUNCTIONAL THEORY TO THE DROPLET-VAPOR SYSTEM OF SIMPLE FLUIDS}

Although Eqs. (12) and (13) are rigorous thermodynamic expressions for the surface tension $\sigma_{s}$ and the radius $R_{s}$ of the surface of tension, the determination of $\sigma_{s}$ and $R_{s}$ requires values of $\Delta \Omega$ (the work of droplet formation) and $\Delta p$. Thermodynamics does not provide these data for the droplet-vapor system; a molecular-based approach (statistical mechanics or computer simulation) is needed to determine them. The density-functional theory of nucleation is such an approach. ${ }^{14,15}$ This approach has the advantage that molecular-level detail can be incorporated, and in the limit of large droplets the theory goes naturally to the classical nucleation theory. Effects of curvature dependence of the surface tension arise naturally, rather than being added as ad hoc assumptions. Using the density-functional theory, one can obtain the density profile $\rho(\mathbf{r})$ of a spherical droplet, which is a critical nucleus of the liquid phase, and also the grand potential $\Omega$ of the droplet-vapor system. ${ }^{14}$

The formalism of the density-functional theory of nucleation can be briefly summarized as follows: The Helmholtz free energy functional is taken to be a simple form ${ }^{17}$

$$
\begin{aligned}
F[\rho(\mathbf{r})]= & \int d \mathbf{r} f_{h}[\rho(\mathbf{r})] \\
& +\frac{1}{2} \iint d \mathbf{r} d \mathbf{r}^{\prime} \rho(\mathbf{r}) \rho\left(\mathbf{r}^{\prime}\right) \phi_{\mathrm{att}}\left(\left|\mathbf{r}-\mathbf{r}^{\prime}\right|\right),
\end{aligned}
$$

where $f_{h}(\rho)$ is the Helmholtz free energy density (the free energy per unit volume) of a uniform hard-sphere fluid, which is given by

$$
f_{h}(\rho)=\rho \mu_{h}(\rho)-p_{h}(\rho) .
$$

The chemical potential $\mu_{h}(\rho)$ and the pressure $p_{h}(\rho)$ of the reference hard sphere fluid are evaluated using the highly accurate Carnahan-Starling equation. ${ }^{18} \phi_{\text {att }}$ in Eq. (18) is the attractive part of a pair potential.

We consider the Lennard-Jones (LJ) fluid and Yukawa fluid in this study. In the case of the LJ fluid, $\phi_{\text {att }}$ is taken to be the Weeks-Chandler-Anderson (WCA) perturbation part of the LJ potential function ${ }^{19}$

$$
\begin{aligned}
\phi_{\mathrm{att}}(r) & =4 \epsilon_{\mathrm{LJ}}\left[\left(\frac{\sigma_{\mathrm{LJ}}}{r}\right)^{12}-\left(\frac{\sigma_{\mathrm{LJ}}}{r}\right)^{6}\right] \quad r \geqslant 2^{1 / 6} \sigma_{\mathrm{LJ}} \\
& =-\epsilon_{\mathrm{LJ}} \quad r<2^{1 / 6} \sigma_{\mathrm{LJ}} .
\end{aligned}
$$

The two parameters $\epsilon_{\mathrm{LJ}}$ and $\sigma_{\mathrm{LJ}}$ are, respectively, the depth of the potential well and the collision diameter. Reduced variables [denoted by an asterisk $(*)$ ] are defined in terms of parameters: distance is given in units of $\sigma_{\mathrm{LJ}}$, energy in units of $\epsilon_{\mathrm{LJ}}$, and temperature in units of $\epsilon_{\mathrm{LJ}} / k_{B}$, where $k_{B}$ is the Boltzmann constant. We chose the LJ droplet-vapor system since it has been extensively studied both by computer simulation $^{9-11}$ and by the density-functional theory. ${ }^{15}$ In order to fairly reproduce the liquid density of the LJ fluid at temperatures lower than the critical point, we use the temperature-dependent hard sphere diameter $d(T) .{ }^{20}$ In the case of the Yukawa fluid, the attractive part is taken to be

$$
\phi_{\text {att }}(r)=-\alpha \lambda^{3} \exp (-\lambda r) / 4 \pi \lambda r .
$$

Furthermore we set $\lambda$ to be $d^{-1}$, the inverse of the hard sphere diameter of the repulsive part. In this system, reduced variables are defined in terms of $d$ and $k_{B} T_{c}$ : distance is given in units of $d$, densities in units of $d^{-3}$, energy in units of $k_{B} T_{c}$, temperature in units of $T_{c}$.

We note that Eq. (18) is based on two key approximations: First, the pair distribution function $\rho^{(2)}\left(\mathbf{r}, \mathbf{r}^{\prime}\right)$ of an inhomogeneous fluid is replaced by $\rho(\mathbf{r}) \rho\left(\mathbf{r}^{\prime}\right)$. This is the so-called random phase approximation. ${ }^{21}$ Second, the local density approximation is used to determine the hard sphere part of the free energy functional. These two approximations have been well tested for weakly inhomogeneous systems such as a vapor-liquid interface and are fairly good approximations when the system is not as close to the critical point.

The grand potential functional is given as a Legendre transform of $F$

$$
\Omega[\rho]=F[\rho]-\mu \int d \mathbf{r} \rho(\mathbf{r})
$$


The density profile of a critical nucleus at $\mu$ and $T$ (as well as that of a planar surface at $\mu_{\mathrm{eq}}$ and $T$ ) can be determined from the equilibrium condition

$$
\delta \Omega[\rho] / \delta \rho(\mathbf{r})=0,
$$

or

$$
\mu=\mu_{h}[\rho(\mathbf{r})]+\int d \mathbf{r}^{\prime} \phi_{\text {att }}\left(\left|\mathbf{r}-\mathbf{r}^{\prime}\right|\right) \rho\left(\mathbf{r}^{\prime}\right) .
$$

We also note that the density profile of the planar surface can be obtained from Eq. (24) using the conventional iteration technique. However, the density profile of a critical nucleus is not a stable solution of Eq. (24) since it corresponds to a saddle point in the functional space. Nonetheless, if an appropriate initial guess is made, one can find the unstable equilibrium density profile which satisfies the condition (23) over many iterations. ${ }^{15}$

The density profile and the grand potential of the critical droplet-vapor system were calculated over a wide range of supersaturation $\Delta \mu \equiv \mu-\mu_{\text {eq }}$ at a fixed temperature: for the LJ system $T^{*}=0.7$, which is close to the triple-point temperature $T_{\mathrm{tp}}^{*} \approx 0.69$ of the system; for the Yukawa system $T / T_{c}=0.6$.

\section{RESULTS AND DISCUSSION}

\section{A. Behavior of the length $\delta$}

The usual way of determining the length $\delta$ is to calculate $R_{e}$ and $R_{s}$ and then use the definition $\delta \equiv R_{e}-R_{s}$, which we hereafter refer to as "a direct route to $\delta$ ". The radius $R_{e}$ of the equimolar surface can be determined from the density profile of a critical droplet by the exact relation

$$
R_{e}^{3}=\frac{3}{\Delta \rho} \int_{0}^{\mathscr{B}}\left[\rho(r)-\rho^{\beta}\right] r^{2} d r .
$$

Location of the surface of tension, $R_{s}$, is given by Eq. (13).

Another way of determining $\delta$, which we propose here, is to use the relation

$$
-\frac{\left(\partial \sigma_{s} / \partial \mu\right)_{T}}{\Delta \rho}=\delta\left(1+\frac{\delta}{R_{s}}+\frac{1}{3} \frac{\delta^{2}}{R_{s}^{2}}\right) .
$$

This is derived from Eqs. (14) and (16). $\sigma_{s}$ and $R_{s}$ are given by the exact expressions (12) and (13), respectively. The derivative $\left(\partial \sigma_{s} / \partial \mu\right)_{T}$ can be calculated via numerical differentiation. We refer to this method as "an adsorption route to $\delta . "$

We now discuss both the result of the direct route and that of the adsorption route. Figure 1 shows $\delta$ of the LJ droplet as a function of supersaturation $\Delta \mu$. When supersaturation is large $\left(\Delta \mu^{*}>0.2\right)$, the behavior of $\delta$ obtained from the two routes is almost indistinguishable. In this range $\delta$ decreases linearly with the decrease in $\Delta \mu$ and changes its sign at about $\Delta \mu^{*}=0.3$. The two routes, however, lead to qualitatively different results when $\Delta \mu^{*}<0.2$, that is, in a range of very large droplets; $\delta$ via direct route turns to be positive and tends to diverge as $\Delta \mu$ goes to zero, whereas $\delta$ via the adsorption route still decreases linearly and eventually converges to a small negative value $\delta_{\infty}=-0.15 \sigma_{\mathrm{LJ}}$ in the planar surface limit. Qualitatively the same result is

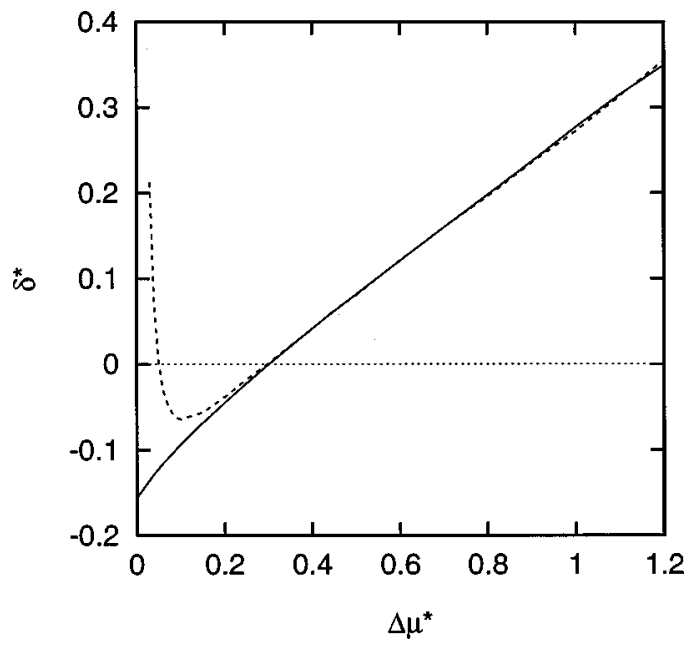

FIG. 1. The length $\delta^{*}$ vs the supersaturation $\Delta \mu^{*}$ at $T^{*}=0.7$ : the direct route (dotted line) and the adsorption route (solid line). The reduced dimensionless variables are defined in terms of the LJ size and energy parameters.

found for the Yukawa potential system; the direct route gives rise to the diverging behavior of $\delta$, whereas the adsorption route leads to $\delta_{\infty}=-0.26 d$. The diverging behavior of $\delta$ via direct route is unphysical because both the equimolar surface $\left(R_{e}\right)$ and the surface of tension $\left(R_{s}\right)$ should lie within the vapor-liquid interface whose width remains finite over a whole range of supersaturation at thermodynamic states near the triple point.

In principle, both direct and adsorption routes should lead to the same behavior of $\delta$ over a whole range of $\Delta \mu$ because no approximations are made in either route (except common approximations in the density-functional theory). Therefore, the problem with the direct route (unphysical behavior of $\delta$ in a range of small $\Delta \mu$ ) is a numerical one rather than a theoretical one. The reason is the following. As $\Delta \mu$ goes to zero, $R_{s}$ and $R_{e}$ increase simultaneously and diverge ultimately. In this process, absolute numerical errors of $R_{s}$ and $R_{e}$ also increase systematically although relative errors may remain small [see, for example, expression (13) for $R_{s}$; the denominator $\Delta p$ goes to zero.] Consequently, relative errors of $\delta$ given by subtracting $R_{s}$ from $R_{e}$ are expected to be large and increase systematically. This problem would be even more serious for computer simulation studies due to the statistical errors. In fact, computer simulation results show that the sign of $\delta$ is uncertain for large droplets. ${ }^{11}$ Thus, we must conclude that exact asymptotic behavior of $\delta$ in the range of very large droplets cannot be obtained through the direct route.

On the other hand, the adsorption route is free from the numerical problem. The reason is the following. This approach requires $\left(\partial \sigma_{s} / \partial \mu\right)_{T}, \Delta \rho$, and $R_{s}$; however, in the large droplet limit the ratio of the first two quantities determines $\delta$ [see Eq. (26)]. Unlike $R_{e}$ and $R_{s}$, both $\left(\partial \sigma_{s} / \partial \mu\right)_{T}$ and $\Delta \rho$ remain finite values as $\Delta \mu$ goes to zero. Thus, the ratio $\left(\partial \sigma_{s} / \partial \mu\right)_{T} / \Delta \rho$ can be determined accurately over a whole range of droplet sizes. As a consequence, asymptotic behavior of $\delta$ and the value of $\delta_{\infty}$ can be determined without suffering from any numerical problem.

Furthermore, it can be seen from the following that the 


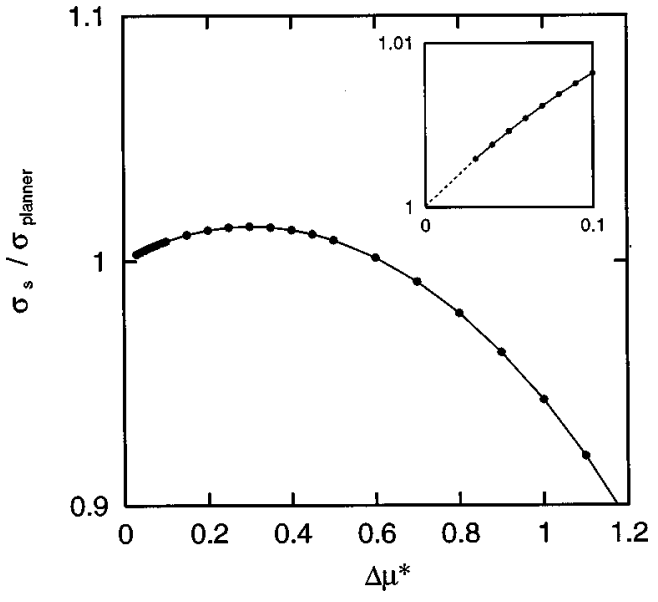

FIG. 2. The surface tension ratio $\sigma_{s} / \sigma_{\infty}$ vs the supersaturation $\Delta \mu^{*}$ at $T^{*}=0.7$. The inset is the magnification at small supersaturation. Dotted line in the inset is drawn by extrapolation.

asymptotic behavior of $\delta$ obtained by the adsorption-route calculation is consistent with the properties of the planar liquid-vapor interface. Figure 2 shows that the surface tension of the spherical surface of the LJ droplets is fully consistent with that of the planar surface; extrapolation shows $\sigma_{s}(\Delta \mu=0) / \sigma_{\infty}=1.00007$, where $\sigma_{\infty}$ was obtained from the density-functional calculation for the planar liquid-vapor interface. The self-consistency for the values of $\sigma_{s}$ guarantees that for $\left(\partial \sigma_{s} / \partial \mu\right)_{T}$. In addition, the behavior of $\Delta \rho$ is also self-consistent. Therefore, the asymptotic behavior of $\delta$ is also consistent with the properties of the liquid-vapor interface within the framework of the density-functional theory.

\section{B. Range of the validity of Tolman's equation}

Once Tolman's length $\delta_{\infty}$ is determined accurately, direct comparison can be made between the behavior of $\sigma_{s}\left(R_{s}\right)$ given by exact thermodynamic relation (12) and that by Tolman's equation (1). Figure 3(a) shows a comparison for the LJ droplet-vapor system. When the reduced radius $R_{s}^{*}$ is greater than about 50, prediction of Tolman's equation is almost indistinguishable in the scale of the plots from the result of the exact thermodynamic formula; the ratio $\sigma_{s}\left(R_{s}\right) / \sigma_{\infty}$ is slightly larger than unity and increases very slowly as $R_{S}$ decreases. The same result was obtained in the case of the Yukawa potential system [Fig. 3(b)]. This confirms that $\delta_{\infty}$ was determined accurately in such a way that $\delta_{\infty}$ is consistent with the asymptotic behavior of $\sigma_{s}$.

However, when $R_{s}^{*}$ is smaller than about 20 , or $N^{\alpha}$ $<10^{6}$, prediction of Tolman's equation becomes significantly different from the result of the rigorous calculation. The surface tension $\sigma_{s}$ of the exact formula shows nonmonotonic behavior; as $R_{s}$ decreases it reaches a maximum at about $R_{s}^{*}=10$ and begins to decrease rapidly. On the other hand, $\sigma_{s}$ of Tolman's equation is necessarily a monotonic function of $R_{s}$; Fig. 3(a) shows monotonic increase in $\sigma_{s}$ with decrease in $R_{s}$ due to the negative value of $\delta_{\infty}$. The same significant difference was found in this range of droplet size in the case of the Yukawa potential fluid [see Fig. 3(b)].
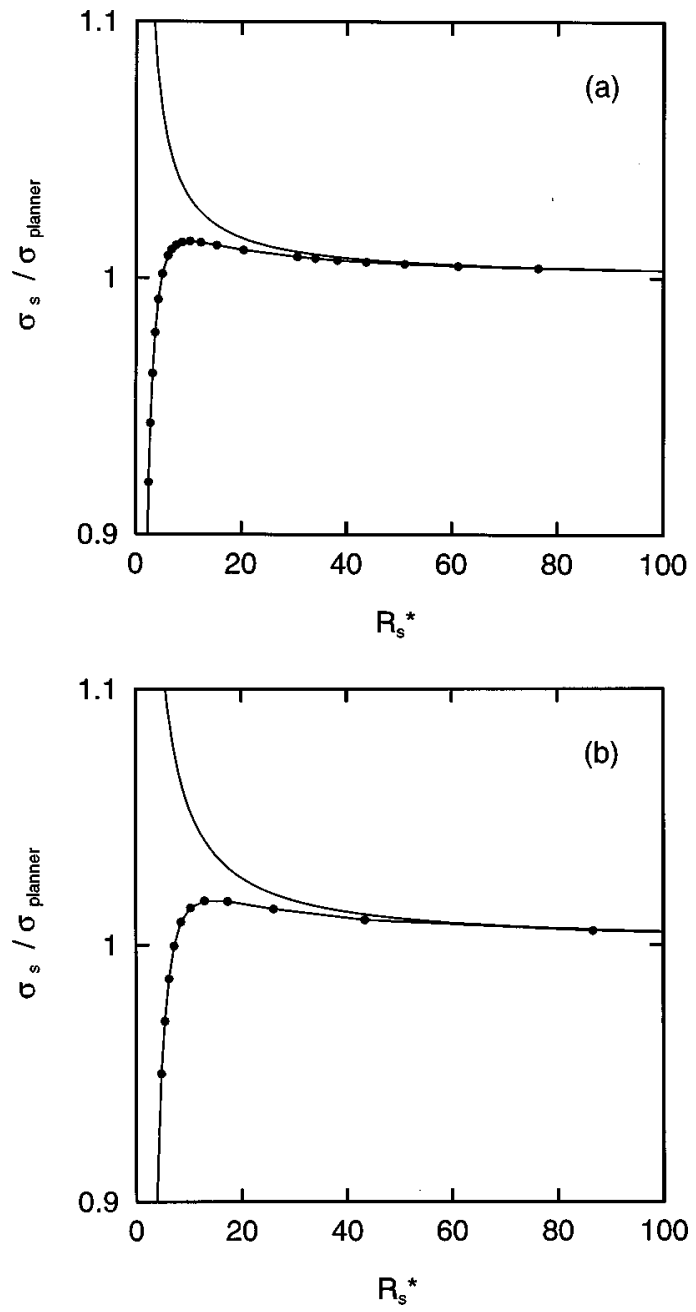

FIG. 3. The surface tension ratio $\sigma_{s} / \sigma_{\infty}$ obtained via the rigorous thermodynamic route (solid line with circles) and the prediction of Tolman's equation (solid line): (a) Lennard-Jones fluid and (b) Yukawa fluid. Values of $\delta_{\infty}$ (in Tolman's equation) were obtained via the adsorption route.

These results suggest that Tolman's equation is valid only for droplets with size $N^{\alpha} \geqslant 10^{6}$, at least near the triple points of the systems. This is the main conclusion of this article. Since basically the same results are obtained from the two distinctive potential functions (LJ and Yukawa), it is very unlikely that replacement of the long-range interaction by $r^{-7}$ (i.e., consideration of the retardation effect in the dispersion force at a very large distance ${ }^{22,23}$ ) would give a qualitatively different result.

We also note that assumption B $\left(\delta=\delta_{\infty}\right)$ becomes incorrect in a range of droplet sizes where assumption $\mathrm{A}\left(\delta / R_{s}\right.$ $\ll 1)$ is still valid; $\delta$ is no longer constant when $R_{s}^{*}<20$, whereas $\delta / R_{s}=0.02$ at $R_{s}^{*}=5$ (see the solid line with points in Fig. 5). The breakdown of assumption B is closely related to the nonmonotonic size dependence of surface tension since if $\delta$ changes its sign so does the term $\left(\partial \sigma_{s} / \partial \mu\right)_{T}$ and vice versa [see Eq. (26)]. Also if Tolman's length $\delta_{\infty}$ is negative, assumption B should become incorrect in some range of droplet sizes since $\delta>0$ and $\left(\partial \sigma_{s} / \partial \mu\right)_{T}<0$ for very small droplets. Thus, $\delta_{\infty}<0$ is also closely related to the breakdown of assumption B and to the nonmonotonic behavior of $\sigma_{s}$. 


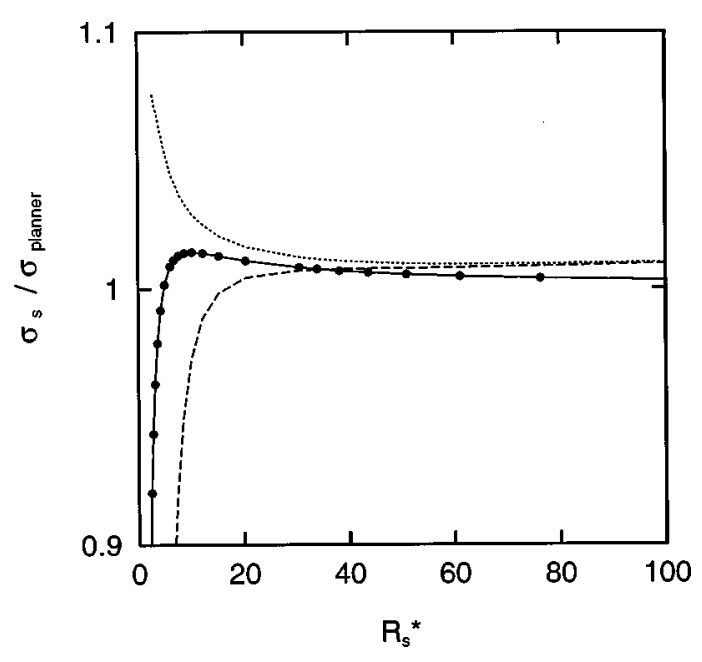

FIG. 4. The surface tension ratio $\sigma_{s} / \sigma_{\infty}$ vs $R_{s}^{*}$ : the rigorous thermodynamic route (solid line with circles); the route of Thompson et al. (dashed line); and the modified route (dotted line).

\section{Examination of other thermodynamic routes}

As mentioned in Sec. III, rigorous calculation of $\sigma_{s}$ and $R_{s}$ requires the work of formation of a critical droplet $\Delta \Omega$ and the pressure difference $\Delta p$ as thermodynamic inputs. Although the latter two quantities can be obtained straightforwardly by the density-functional theory, they are not easily obtained from computer simulation. For example, the equation of state of the bulk fluid is required to determine the pressures $\left(p^{\alpha}\right.$ and $\left.p^{\beta}\right)$ of the reference homogeneous system unless the droplet size is sufficiently large so that $p^{\alpha}$ can be replaced by the value at the droplet center $p(0)$.

To circumvent the technical difficulties in the simulation, several alternatives to the rigorous thermodynamic routes have been proposed. Since these thermodynamic routes involve certain approximations, the extent to which they are applicable for determining $\sigma_{s}$ as a function of droplet size needs to be examined. One thermodynamic route we examine here was originally proposed by Thompson et al. in their molecular dynamics study of microliquid drops. ${ }^{10}$ In this route, $\sigma_{s}$ and $R_{s}$ are obtained by solving the two coupled equations: Tolman's equation (1) and Laplace's equation (11). Approximations in this route are the following: (i) the use of Tolman's equation; ${ }^{24}$ (ii) replacement of the constant $\delta_{\infty}$ in Eq. (1) by a variable $\delta=R_{e}-R_{s}$; (iii) replacement of $\Delta p$ in the rigorous thermodynamic relation (11) by $\Delta p(0)$; and (iv) replacement of $\Delta \rho$ in the formula (25) by $\Delta \rho(0)$ $\equiv \rho(0)-\rho^{\beta}$. Note that all the approximations listed above turn to be exact in the large droplet limit. This route is particularly convenient for the computer simulation since quantities such as $R_{e}, \Delta p(0)$, and $\Delta \rho(0)$ can be calculated directly in the simulation of a droplet-vapor system. However, in order to apply this method we need to clarify the range of validity for these approximations.

Figure 4 compares behavior of $\sigma_{s}$ obtained via the route of Thompson et al. with that obtained via the rigorous route over a wide range of droplet sizes. The difference is significant for small droplets, that is, in the range $R_{s}^{*}<20$. Similar results at different temperatures have been obtained by Ta-

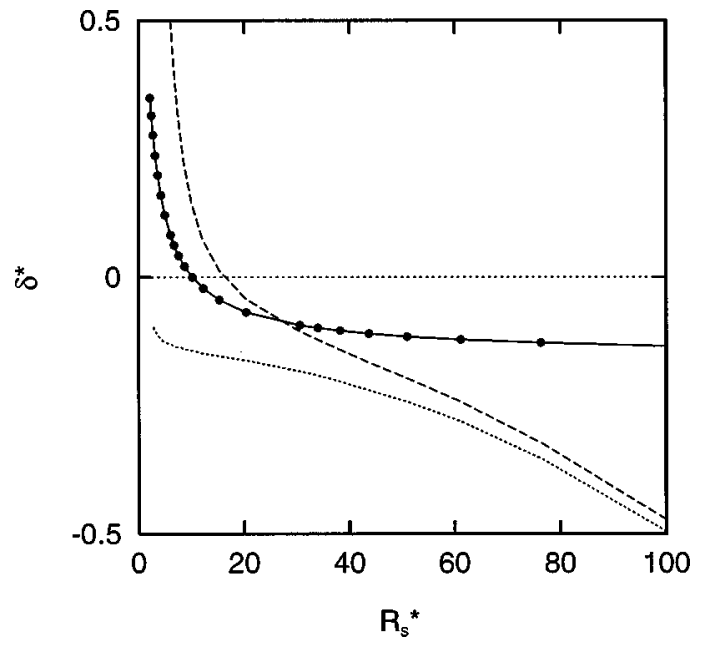

FIG. 5. The length $\delta^{*}$ vs $R_{s}^{*}$ : the strict thermodynamic route (solid line with circles); the route of Thompson et al. (dashed line); and the modified route (dotted line).

lanquer and Oxtoby. ${ }^{7}$ We also find from Fig. 4 that even for very large droplets (e.g., $R_{s}^{*} \sim 100$ where Tolman's equation is valid and all the other approximations are totally reasonable) there exists a small but systematic difference in $\sigma_{s}$. Again, this is due to the fact that the quantity $\delta$ is determined by the subtraction of two large quantities, $R_{e}-R_{s}$, which causes larger and larger numerical errors as we go to larger and larger droplets (see Fig. 5). The computer simulation is more prone to this problem because of the statistical errors involved in averaging.

Let us consider the possibility of improving the thermodynamic route of Thompson et al. Since both the use of Tolman's equation and the replacement of $\delta_{\infty}$ by $\delta$ are key approximations in this route, let us focus on approximations (iii) and (iv), which can be removed provided the equation of state for the particular system is known. It is expected that the effect of approximation (iii) on the results becomes larger and larger with the decrease in droplet size. This is because the size dependence of the center pressure $\Delta p(0)$ is opposite that of $\Delta p$ in a range of small droplets; $\Delta p(0)$ decreases with the decrease in droplet size, whereas $\Delta p$ increases monotonically.

To examine the effects of approximations (iii) and (iv) we calculated $\sigma_{s}$ and the length $\delta$ after replacing $\Delta p(0)$ and $\Delta \rho(0)$ by $\Delta p$ and $\Delta \rho$ of the rigorous route. (We refer to this approach as the modified route.) Figures 4 and 5 show the results of $\sigma_{s}$ and $\delta$ determined by the modified route, together with the results from the rigorous route and from the route of Thompson et al. Contrary to our expectation, we find that results of the modified route are rather worse than those of the original route of Thompson et al. except in the large droplet limit where both results converge to the same values. That is, with the decrease in $R_{s}$ the surface tension increases monotonically and the values of $\delta$ remain negative over a whole range of $R_{s}$. The fact that removing approximations (iii) and (iv) worsens the results of the route of Thompson et al. indicates that although the results obtained via the route of Thompson et al. bear some similarities to those obtained via the rigorous route (e.g., as $R_{s}$ decreases 
$\sigma_{s}$ decreases for a range of small droplets, and $\delta$ changes its sign from negative to positive), these similarities appear fortuitous.

From these analyses, we conclude that as far as the size dependence of the surface tension and Tolman's length $\delta_{\infty}$ are concerned, the thermodynamic route proposed by Thompson et al. seems unsuitable.

Nijmeijer et al. ${ }^{11}$ also attempted to determine $\delta_{\infty}$ from the molecular dynamics simulation of LJ droplets with reduced radii $R_{s}^{*} \leqslant 13$. The route of Nijmeijer et al. to $\delta_{\infty}$ requires the use of the relation

$$
\frac{R_{e} \Delta p(0)}{\sigma_{\infty}}-2=-\frac{2 \delta}{R_{e}},
$$

which entails essentially the same approximations as used in the route of Thompson et al. Thus the route of Nijmeijer et al. should encounter the same problem emerged in the route of Thompson et al., particularly when the droplet is small. Although Eq. (27) becomes exact in the large droplet limit, again this route invokes the subtraction $R_{e}-R_{s}$ which causes large numerical errors for $\delta$. The limitation of this thermodynamic route to $\delta_{\infty}$ has already been pointed out by Nijmeijer et al. ${ }^{11}$

\section{CONCLUDING REMARKS}

Based on the Gibbs thermodynamic theory of capillarity combined with the density-functional theory of gas-liquid nucleation, size dependence of the surface tension of LJ and Yukawa droplets and that of $\delta$ were investigated over a wide range of droplet sizes. Tolman's length $\delta_{\infty}$, namely $\delta$ in the planar surface limit, was determined unequivocally via what we call the adsorption route to $\delta$ which relies on an exact thermodynamic relation (26). Direct comparison between result of rigorous calculation based on the thermodynamic relations (12)-(13) and that from Tolman's equation shows that Tolman's equation is valid only when the droplet holds more than $10^{6}$ molecules or has a radius larger than about 20 molecular diameters, which is much larger than droplets mostly studied so far by computer simulations. We pointed out that Tolman's equation becomes invalid mainly due to the breakdown of assumption B $\left(\delta=\delta_{\infty}\right)$, rather than A ( $\delta$ $\ll R_{s}$ ). As discussed in Sec. IV, the breakdown of assumption B is closely related to the nonmonotonic behavior of $\sigma_{s}$ (as a function of $R_{s}$ or $\Delta \mu$ ) and the negative value of Tolman's length. The nonmonotonic behavior has been found previously in the framework of phenomenological gradient theories ${ }^{4,5}$ and the square gradient theory with a doubleparabola model for free energy density. ${ }^{6}$ In the latter model, Tolman's length is expressed in terms of correlation lengths of bulk gas and liquid phases; Iwamatsu concludes from the result that in general Tolman's length should be negative ( $\delta_{\infty}=-0.38 d$ at $T=0.8 T_{c}$ ). Also, recent calculation of $\delta_{\infty}$ (as the first-order curvature correction to the surface free energy) based on a mean field approximation shows that $\delta_{\infty} \approx-0.20 d$ at all temperatures. ${ }^{25}$ Furthermore it is shown ${ }^{26}$ that close results are obtained from three different formulas (two of Blokhuis and Bedeaux ${ }^{27}$ and one of Bykov and Shchekin) for calculating Tolman's length from the first- order curvature correction to the profile of the planner interface. Since nonmonotonic behavior of surface tension or negative Tolman's length is a common result of all recent mean-field theories with different approximations, it is very unlikely that our results are due to the random phase approximation to the perturbation term in the functional we employed or to the potential functions (LJ and Yukawa) for the attractive part we employed. It is, however, a future subject to examine these results using more sophisticated approximations to the reference hard sphere part such as a nonlocal density functional approximation. ${ }^{28}$

We also discussed to what extent other thermodynamic approaches ${ }^{10,11}$ can be used as alternatives to the rigorous thermodynamic approach for studying the size dependence of surface tension and of the length $\delta$. Although these approximate methods become formally exact in the large droplet limit, correct asymptotic behavior of the surface tension and length $\delta$ cannot be obtained via these routes due to the underlying numerical-error problem in evaluating $\delta$.

The results summarized above suggest that in the application of computer simulation to the investigation of the size dependence of surface tension of a liquid droplet, one should consider the so-called mechanical routes ${ }^{9,16}$ or statistical mechanical routes ${ }^{23,29}$ rather than the thermodynamic routes. The reasons are the following. The strict thermodynamic route to the surface tension requires certain quantities which are not readily calculated from computer simulations. Other alternative thermodynamic routes proposed for computer simulation are not valid for small droplets due to the approximations such as the use of Tolman's equation, and can be problematic for large droplets due to the numerical-error problem. On the other hand, we note that through the statistical mechanical route as proposed by Blokhuis and Bedeaux ${ }^{23}$ computer simulation can be still useful for the calculation of $\delta_{\infty}$. This route demands information on the pair distribution functions of the planar liquid-vapor interface, which can be calculated straightforwardly via molecular simulation. Indeed results of molecular dynamics simulations of Haye and Bruin $^{29}$ do not suffer from the large numerical errors problem as appeared in the simulations based on thermodynamic routes. However, their values of $\delta_{\infty}$ at several temperatures are all positive (e.g., $\delta_{\infty}^{*}=0.16$ at $T^{*}=0.75$ ), which contrast with our calculation based on the combined theory that $\delta_{\infty}$ is negative near the triple point ( $\delta_{\infty}=-0.15 \sigma_{\mathrm{LJ}}$ at $T=0.70 \epsilon_{\mathrm{LJ}} / k_{B}$ for the LJ fluid; $\delta_{\infty}$ $=-0.26 d$ at $T=0.6 T_{c}$ for the Yukawa fluid). As mentioned above other mean-field or density-functional approaches ${ }^{5,6,25,26}$ also give rise to negative values of $\delta_{\infty}$. Further investigation is needed to resolve the difference in prediction of the sign from the simulation and from the theoretical approaches.

The density-functional theory of nucleation when combined with the strict thermodynamic route to the surface tension is shown to be a very useful tool for examining the size dependence of surface tension and of length $\delta$ of a critical droplet. Despite the fact that the absolute value of the surface tension is dependent on the underlying approximations (such as the local density approximation), this combined theory has the virtue of self-consistency in calculation over a whole 
range of droplet sizes, including the planar surface limit. Consequently, the density-functional theory provides a unique means for determining Tolman's length. Nevertheless, the elusiveness of its sign still demands further studies.

\section{ACKNOWLEDGMENTS}

The authors are grateful to Dr. D. J. Diestler for helpful discussions. XCZ thanks the NSF and ONR for support of this work. AKS also thanks RFBR (Grant No. 96-02-18959) for support of this work.

${ }^{1}$ J. W. Gibbs, The Scientific Papers of J. Willard Gibbs (Ox Bow, Woodbridge, NJ, 1993).

${ }^{2}$ R. C. Tolman, J. Chem. Phys. 17, 333 (1949).

${ }^{3}$ A. H. Falls, L. E. Scriven, and H. T. Davis, J. Chem. Phys. 75, 3986 (1981).

${ }^{4}$ R. Guermeur, F. Biquard, and C. Jacolin, J. Chem. Phys. 82, 2040 (1985).

${ }^{5}$ V. G. Baidakov and G. Sh. Boltachev, Russ. J. Phys. Chem. 69, 468 (1995).

${ }^{6}$ M. Iwamatsu, J. Phys.: Condens. Matter 6, L173 (1994).

${ }^{7}$ V. Talanquer and D. W. Oxtoby, J. Chem. Phys. 99, 2865 (1995).

${ }^{8}$ A. Laaksonen and R. McGraw, Europhys. Lett. 35, 367 (1996).

${ }^{9}$ A. I. Rusanov and E. N. Brodskaya, J. Colloid Interface Sci. 62, 542 (1977).

${ }^{10}$ S. M. Thompson, K. E. Gubbins, J. P. R. B. Walton, R. A. R. Chantry, and J. S. Rowlinson, J. Chem. Phys. 81, 530 (1984).

${ }^{11}$ M. J. P. Nijmeijer, C. Bruin, A. B. van Woerkom, A. F. Bakker, and J. M. J. van Leeuwen, J. Chem. Phys. 96, 565 (1992).

${ }^{12}$ E. N. Brodskaya, J. C. Eriksson, A. Laaksonen, and A. I. Rusanov, J. Colloid Interface Sci. 180, 86 (1996).
${ }^{13}$ In fact, Tolman correctly noted in the discussion of the validity of Eq. (1) that $\delta$ can be expected to vary appreciably when droplets are small enough. Also Tolman predicted the rapid decrease in surface tension; the conclusion was, however, derived from Eq. (1).

${ }^{14}$ D. W. Oxtoby and R. Evans, J. Chem. Phys. 89, 7521 (1988).

${ }^{15}$ X. C. Zeng and D. W. Oxtoby, J. Chem. Phys. 94, 4472 (1991).

${ }^{16} \mathrm{~J}$. S. Rowlinson and B. Widom, Molecular Theory of Capillarity (Oxford, New York, 1989).

${ }^{17}$ P. Tarazona and R. Evans, Mol. Phys. 48, 799 (1983).

${ }^{18}$ N. F. Carnahan and K. E. Starling, J. Chem. Phys. 51, 635 (1969).

${ }^{19}$ J. D. Weeks, D. Chandler, and H. C. Anderson, J. Chem. Phys. 54, 5237 (1971).

${ }^{20}$ B. Q. Lu, R. Evans, and M. M. Telo da Gama, Mol. Phys. 55, 1913 (1985).

${ }^{21}$ R. Evans, Adv. Phys. 28, 143 (1979).

${ }^{22}$ J. S. Rowlinson, J. Phys.: Condens. Matter 6, A1 (1994).

${ }^{23}$ E. M. Blokhuis and D. Bedeaux, J. Chem. Phys. 97, 3576 (1992).

${ }^{24}$ Strictly speaking, Thompson et al. used the truncated form of Tolman's equation in their calculation; we used the original form of Tolman's equation. However, we confirmed that our conclusion does not depend on the choice.

${ }^{25}$ A. E. van Giessen, E. M. Blokhuis, and D. J. Bukman, J. Chem. Phys. 108, 1148 (1998).

${ }^{26}$ V. G. Bykov and A. K. Shchekin, in Proceedings of International Workshop, "Nucleation and Non-linear Problems in the First-Order Phase Transition,', St. Petersburg, 1998.

${ }^{27}$ E. M. Blokhuis and D. Bedeaux, Physica A 184, 42 (1992).

${ }^{28}$ W. E. Brown, R. J. F. Decarvalho, and R. Evans, Mol. Phys. 88, 579 (1996).

${ }^{29}$ M. J. Haye and C. Bruin, J. Chem. Phys. 100, 556 (1994). 